\title{
Depression and frailty in later life: a systematic review
}

This article was published in the following Dove Press journal:

Clinical Interventions in Aging

15 December 2015

Number of times this article has been viewed

\section{Leslie Vaughan' \\ Akeesha L Corbin' \\ Joseph S Goveas ${ }^{2}$}

'Department of Social Sciences and Health Policy, Wake Forest School of Medicine, Winston-Salem, NC, USA; ${ }^{2}$ Department of Psychiatry and Behavioral Medicine, Medical College of Wisconsin, MI, USA
Correspondence: Leslie Vaughan Department of Social Sciences and Health Policy, Wake Forest University School of Medicine, Medical Center Boulevard, Winston-Salem, NC 27I57, USA Email alvaugha@wakehealth.edu

\begin{abstract}
Frailty and depression are important issues affecting older adults. Depressive syndrome may be difficult to clinically disambiguate from frailty in advanced old age. Current reviews on the topic include studies with wide methodological variation. This review examined the published literature on cross-sectional and longitudinal associations between frailty and depressive symptomatology with either syndrome as the outcome, moderators of this relationship, construct overlap, and related medical and behavioral interventions. Prevalence of both was reported. A systematic review of studies published from 2000 to 2015 was conducted in PubMed, the Cochrane Database of Systematic Reviews, and PsychInfo. Key search terms were "frailty", "frail", "frail elderly", "depressive", "depressive disorder", and "depression". Participants of included studies were $\geq 55$ years old and community dwelling. Included studies used an explicit biological definition of frailty based on Fried et al's criteria and a screening measure to identify depressive symptomatology. Fourteen studies met the inclusion/exclusion criteria. The prevalence of depressive symptomatology, frailty, or their co-occurrence was greater than $10 \%$ in older adults $\geq 55$ years old, and these rates varied widely, but less in large epidemiological studies of incident frailty. The prospective relationship between depressive symptomatology and increased risk of incident frailty was robust, while the opposite relationship was less conclusive. The presence of comorbidities that interact with depressive symptomatology increased incident frailty risk. Measurement variability of depressive symptomatology and inclusion of older adults who are severely depressed, have cognitive impairment or dementia, or stroke may confound the frailty syndrome with single disease outcomes, accounting for a substantial proportion of shared variance in the syndromes. Further study is needed to identify medical and behavioral interventions for frailty and depressive symptomatology that prevent adverse sequelae such as falls, disability, and premature mortality.
\end{abstract}

Keywords: frailty, depression, depressive symptomatology, aging

\section{Introduction}

The frailty phenotype was defined by Fried et al ${ }^{1}$ as "a biologic syndrome of decreased reserve and resistance to stressors, resulting from cumulative declines across multiple physiologic systems, and causing vulnerability to adverse outcomes" including falls, hospitalization, disability, and death. It was first operationalized and measured over 3 and 7 years in noninstitutionalized women and men of ages 65-101 years in the Cardiovascular Health Study at baseline as $\geq 3$ of the following criteria present: unintentional weight loss of $\geq 10 \mathrm{lbs} / \geq 5 \%$ of body weight accompanied by sarcopenia in the prior year; weakness (grip strength), slowness (walking time), low activity (kcals per week) in the 20th percentile; and self-reported "exhaustion" from two items on the Center for Epidemiological Studies Depression (CES-D) Scale. ${ }^{2}$ The initial study excluded those with a history of probable dementia, stroke, mini-mental scores $<18$, and taking antidepressants, the rationale being that "these conditions could potentially 
present with frailty characteristics as a consequence of a single disease". The original findings supported the hypothesized physiologic "cycle of frailty", "stages" of frailty, "reversibility" of frailty, and that frailty was greater in women and not synonymous with either disability or comorbidity. Other distinct conceptual models of frailty have also been proposed, which are extensively reviewed elsewhere and will not be discussed here. ${ }^{3-5}$ Frailty as a phenotype has significant public health relevance because its identification in the earliest stages may prevent falls, hospitalizations, disability, and premature death.

Recent interest in "overlapping syndromes" in general but more specifically the concept of overlap in frailty and depression - has spawned reviews indicating a positive association between the two. ${ }^{6,7}$ This is not surprising given that the frailty phenotype and depression share "exhaustion" criteria from a measure of depression at the outset. Examining the relationship between frailty and depression is further complicated by the confound between frailty and disability, because the latter two constructs also "overlap" but are distinct. ${ }^{1,89}$ Depression as a "syndrome", or set of medical signs and symptoms that are correlated with each other, may be difficult to disambiguate clinically from frailty in advanced old age. For example, depression and frailty share presenting symptoms such as low daily activity profiles, which could result from either decreased energy reserve (in both frailty and depression) and loss of interest (anhedonia in depression), or loss of ability to engage in daily life activities (disability). Further, a "cycle" of frailty leading to falls, hospitalization, disability, or death is a causal conundrum because individuals can present at any time anywhere in the process. For instance, an individual with a lifelong disability can become frail at an age of 65 years because of decreased physiologic reserve, or an 85-year-old woman who trips, falls, and is hospitalized for hip fracture can become deconditioned and thus "exhausted" because she cannot participate in her normal daily routines, which can subsequently lead to depression and frailty.

Although similar biological mechanisms have been hypothesized to account for both syndromes - frailty and depression - for example, subclinical cardiovascular disease $^{10,11}$ and inflammation, ${ }^{1,12,13}$ it is unlikely that one mechanism is largely responsible for either or both syndromes in all individuals. In fact, although the overlap between depression and frailty is underexplored, evidence to date demonstrates that they are distinct constructs that share moderate overlap (similar to frailty and disability). For example, Mezuk et a ${ }^{14}$ used confirmatory latent class analysis and found that frailty and depression represented distinct constructs (a three-class solution for depression and a two-class solution for frailty with moderate correspondence between the two $[\kappa=0.66 ; 95 \%$ confidence interval $\{C I\}=0.58-0.74]$ ). While many constructs are distinct yet share substantial overlap (as in the case of the frailty and depression constructs), it may be useful to ask the following questions: Are the distinctions between these two syndromes clinically relevant, and if so, why should we care if they are 'overlapping constructs'? Is there some 'common cause' that could be the target of a specific medical or behavioral intervention? The frailty phenotype is undoubtedly still the topic of lively debate, and although experts agree on a more comprehensive definition of frailty that includes, among other things, the assessment of mental health, it is also important to note that there is still no consensus as to the clinical presentation of frailty for practical screening purposes. ${ }^{15}$ Limitations of extant reviews on frailty and depression are the few studies included $(<40) ;^{6-7}$ no rigorous classification of depression; ${ }^{7}$ no use of Fried criteria; ${ }^{6}$ confounding disability as an outcome with frailty; ${ }^{6}$ no published effect sizes, ${ }^{6,7}$ and a lack of longitudinal cohort studies that explore uni- or bidirectional causal relationships between frailty and depression. This review addressed the previously mentioned limitations by including large epidemiological studies with high-quality protocols and well-defined constructs in order to minimize the methodological variance across studies, a common concern in the frailty literature. ${ }^{15}$

\section{Methods}

\section{Data sources and study selection}

A systematic electronic search of studies published from 2000 to 2015 was conducted in MEDLINE, the Cochrane Database of Systematic Reviews, and PsychInfo with the assistance of a Reference Librarian for articles on depression and frailty. Key Medical Subject Headings (MeSH) search terms used in MEDLINE and Cochrane Database included frailty or frail or frail elderly and depressive or depressive disorder or depression. The search was further limited by selecting articles including individuals aged 55 years or older and articles in English language that are published or in press. All titles and abstracts of identified articles were screened independently by two researchers for the inclusion and exclusion criteria described in the following section, and the full-text articles were evaluated for potential inclusion by Leslie Vaughan. The reference lists of the included studies were additionally scanned to identify other articles that made a significant contribution to the literature across multiple 
studies. Final agreement was reached by the researchers regarding inclusion of the studies before including them in the results.

\section{Inclusion criteria}

Articles on frailty and depression were included in this review if they were published from 2000 to 2015; in English; the age group was $\geq 55$ years; participants were community-dwelling; depression or depressive symptomatology was identified by a screening measure (typically some version of the CES$\mathrm{D}^{16}$ or Geriatric Depression Scale [GDS], ${ }^{17}$ although we did not exclude other depression screening measures); used an explicit biological definition of frailty based on the Fried et al's $^{1}$ criteria; and included depressive symptomatology or frailty as an outcome. While we included both cross-sectional and longitudinal observational cohort studies, the included studies had to examine both depression and frailty - either as cross-sectional or longitudinal relationships, overlapping constructs, or causal relationships (uni- and bidirectional). The majority of studies included frailty prevalence, but this was not a requirement for inclusion.

\section{Exclusion criteria}

Articles that did not include both depression and frailty, had poorly defined constructs, examined young or middleaged adults, examined frailty secondary to a developmental disability, frailty in hospitalized or institutionalized older adults, or caregivers of frail elderly were excluded from the review, as were frailty assessment/instrument validity studies.

\section{Results}

The search yielded 461 indexed articles and 100 nonindexed (in press) articles in MEDLINE and 57 articles in PsychInfo for a total of 618 articles retrieved by all searches. After removal of duplicates, the titles and abstracts of the 595 remaining articles were screened by Leslie Vaughan and Akeesha Corbin using the inclusion and exclusion criteria described earlier, and 529 articles that did not meet the specified criteria were excluded. This resulted in 66 full-text articles that were next individually screened for potential inclusion. An additional 52 articles were excluded because either depressive symptomatology or frailty was not the main outcome, participants were not community dwelling, inclusion criteria for the depressive symptomatology or frailty definitions were not met, or either depressive symptomatology or frailty was not evaluated in the study, resulting in the inclusion of a total of 14 studies in the results section for the final review (see Figure 1 for additional details). No related intervention studies met the inclusion criteria.

Prevalence estimates (Table 1) in studies examining cross-sectional associations between depressive symptomatology and frailty ranged from $9.3 \%$ to $21.1 \%$ for frailty. ${ }^{14,18-23}$ Depressive symptomatology prevalence was reported in five of the studies and ranged from $11 \%$ to $25.3 \%,{ }^{14,18,20-22}$ with greater variability in depressive symptomatology classification than frailty classification. The prevalence of depressive symptoms in frail participants ranged from $20.7 \%$ to $53.8 \%,{ }^{14,18-22}$ with one study reporting depressive symptoms in frail participants with anemia. ${ }^{23}$

Longitudinal studies reported baseline frailty estimates ranging from $2.5 \%$ to $16.3 \%{ }^{1,24-26}$ and baseline depression estimates ranging from $6.5 \%$ to $21.3 \%{ }^{1,24,25,27,28} \mathrm{New}$ incident frailty, where examined, ranged from $7.2 \%$ to $18.6 \%{ }^{1,26-29}$ New incident depressive symptomatology, where examined, ranged from $7.5 \%{ }^{29}$ to $30.6 \%{ }^{25}$ Their concurrent prevalence at baseline ranged from $16.4 \%$ to $31.0 \%,{ }^{1,24-26}$ in year 2 was $20.9 \%,{ }^{24}$ and in year 3 was $24.1 \%{ }^{26}$

\section{Cross-sectional associations between depressive symptomatology and frailty}

A few of the secondary analyses of large observational cohort studies, whose primary objective was to assess frailty prevalence, have also examined cross-sectional associations between pre-frailty, frailty, and depressive symptomatology. In older adults $(\mathrm{N}=1,933) \geq 60$ years randomly sampled from the SADEM study, Sanchez-Garcia et $\mathrm{al}^{18}$ found higher odds of depression in frail (odds ratio [OR] $=11.23$; 95\% CI $=10.89-11.58)$ and pre-frail (OR $=3.82 ; 95 \%$ $\mathrm{CI}=3.72-3.93$ ) older adults in multinomial logistic regression models compared to non-frail older adults. In older adults $(\mathrm{N}=640) \geq 75$ years sampled from FRALLE study, ${ }^{19}$ depressive symptomatology was also associated with greater odds of frailty in multivariate analyses $(\mathrm{OR}=3.13$; $95 \% \mathrm{CI}=1.37-7.13$ ), but factors associated with pre-frailty were not reported. Using stratified proportion sampling in a sample of 958 older adults $>60$ years living in an urban area in Brazil, Pegorari and Santos Taveres ${ }^{20}$ found that depressive symptomatology was associated with frailty $(\mathrm{OR}=1.80$; 95\% CI $=1.04-3.12)$, but not pre-frailty (OR $=1.07$; 95\% $\mathrm{CI}=0.71-1.61)$; however, cognitive decline was an exclusion criteria in this study in contrast to the former two studies. Finally, in a convenience sample of older adults $\geq 55$ years $(\mathrm{N}=330)$ from a larger study of midlife that excluded those with a dementia diagnosis, depressive symptomatology 


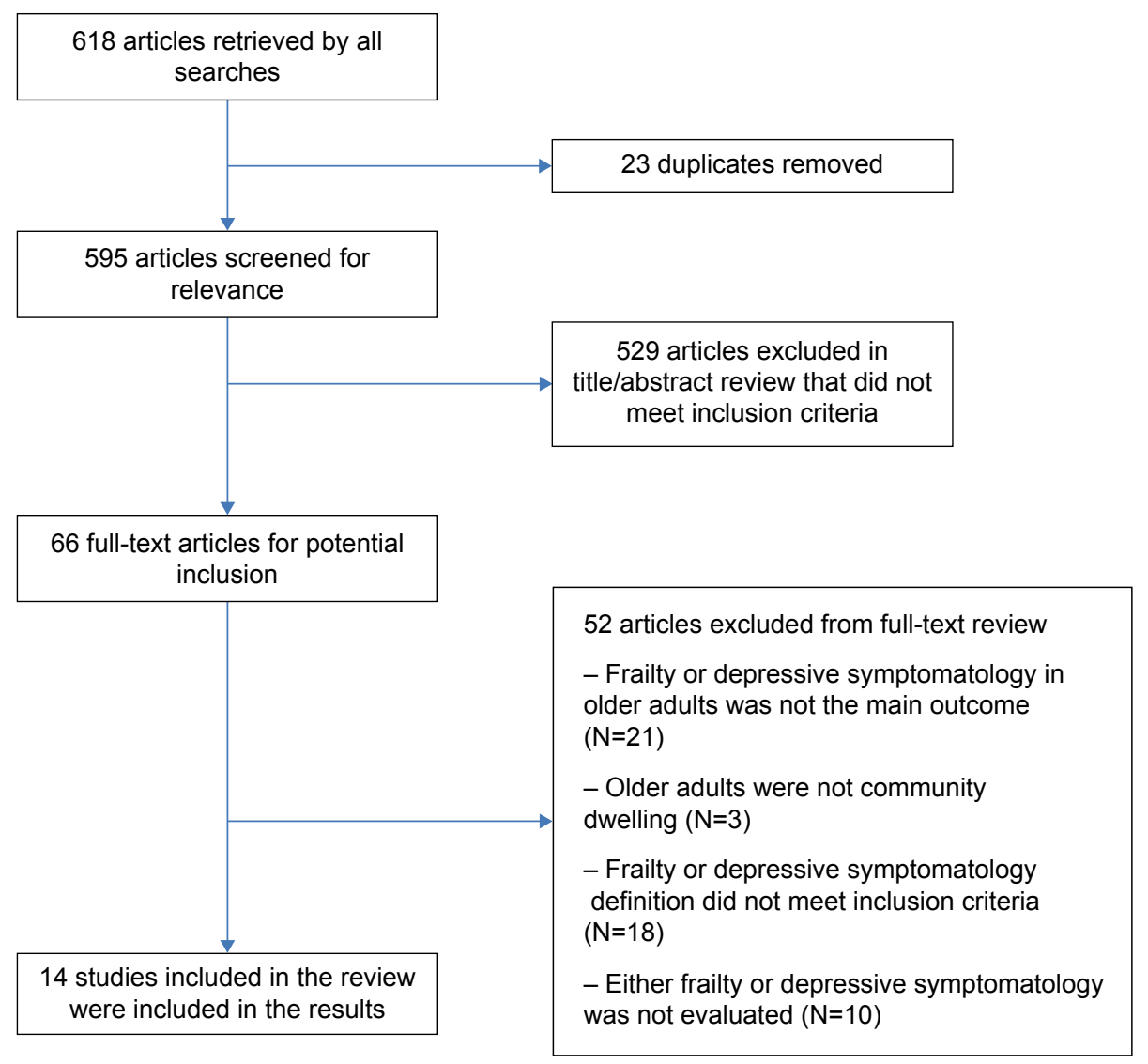

Figure I Search and inclusion strategies.

was significantly associated with frailty $(\mathrm{OR}=4.31 ; 95 \%$ $\mathrm{CI}=1.15-11.9)$ and only marginally associated with prefrailty $(\mathrm{OR}=1.59 ; 95 \% \mathrm{CI}=0.9-2.5) .{ }^{14}$ Although these studies used similar and adequate construct definitions of frailty and depressive symptomatology, not all of them excluded those with a dementia diagnosis.

\section{Longitudinal associations between frailty and depressive symptomatology Depressive symptomatology as a longitudinal predictor of frailty}

In addition to the study ${ }^{1}$ originally linking depressive symptomatology to current (prevalent) frailty and newonset (incident) frailty in older adults, this finding has been replicated in a larger longitudinal cohort study of older women, the Women's Health Initiative (WHI). ${ }^{26}$ Woods et $\mathrm{a}^{26}$ examined frailty at baseline in 40,657 women aged 65-79 and incident frailty 3 years later in 28,181 women who were initially free of frailty at baseline. Adjusted odds of new incident frailty in women with the highest level of depressive symptomatology ( $\geq 5$ on the six-item CES-D) were significantly increased $(\mathrm{OR}=2.20 ; 95 \% \mathrm{CI}=1.88-2.57)$ compared to women with little or no depressive symptomatology. Similarly, the highest levels of depressive symptomatology were associated with increased incidence of intermediate frailty ( $\mathrm{OR}=1.80 ; 95 \% \mathrm{CI}=1.60-2.02)$. A related paper examining the associations between depressive symptomatology, antidepressant use, and incident frailty in the WHI cohort showed that antidepressant users with depressive symptoms were 3.63 times more likely to develop frailty (95\% CI $=2.37-5.55)$, whereas antidepressant nonusers with depressive symptomatology also demonstrated significantly greater odds $(\mathrm{OR}=2.05 ; 95 \% \mathrm{CI}=1.71-2.46)$ compared to nondepressed women. ${ }^{27}$

\section{Frailty as a longitudinal predictor of depressive symptomatology}

A few studies have prospectively examined whether presence or absence of frailty at baseline predicts new-onset incident depressive symptomatology, all with positive findings. In a population-based cohort study - InCHIANTI - of 888 frail older adults aged $\geq 65$ years with follow-up at 3, 6, and 9 years, $30.6 \%$ of nondepressed persons developed depressed mood during follow-up, and the presence of 


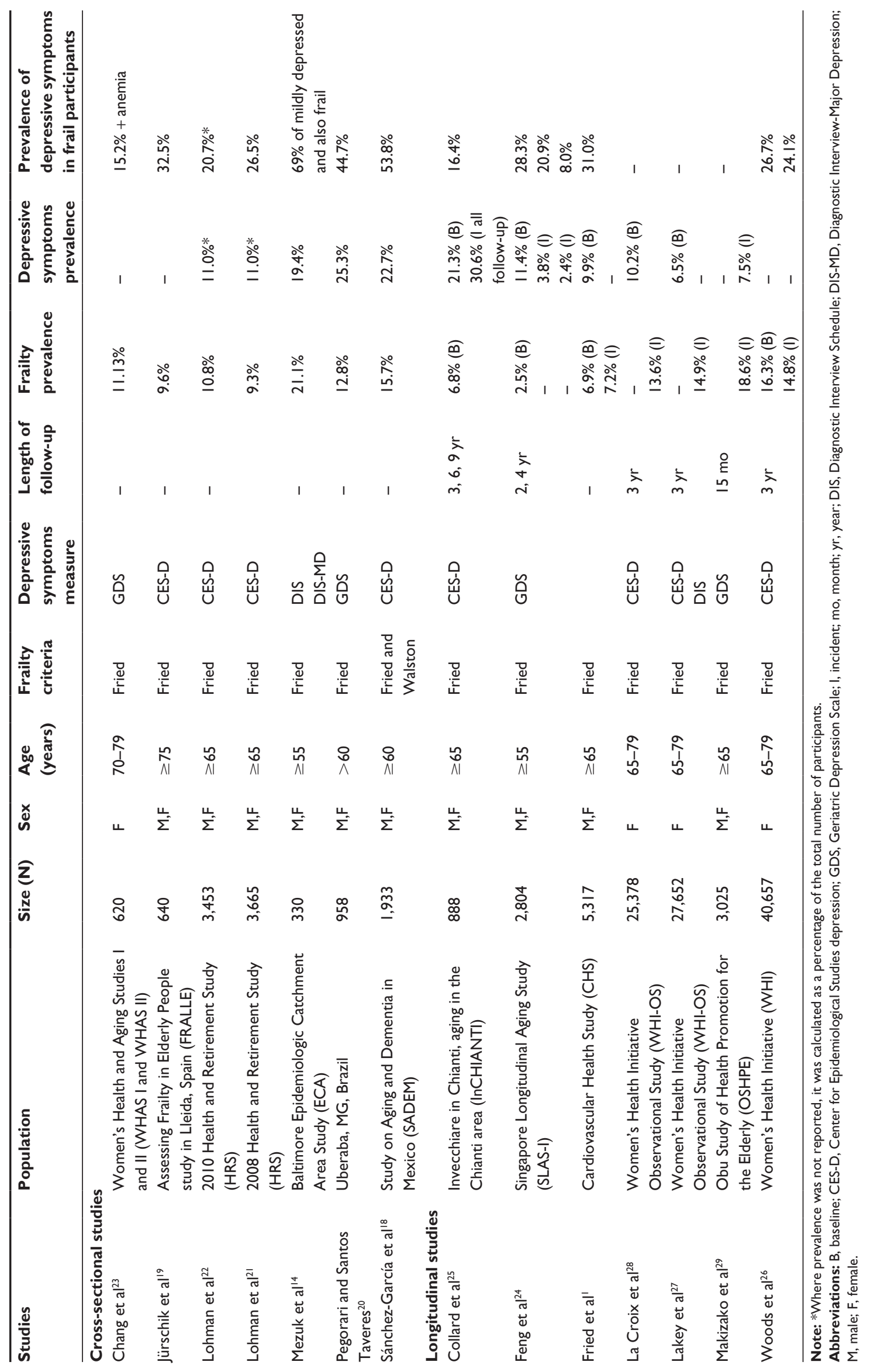


frailty conferred a significant risk of new onset of depression (Hazard ratio $[\mathrm{HR}]=1.26$; 95\% CI $=1.09-1.45$ ) in adjusted models. ${ }^{25}$ Similarly, in the SLAS-I study, ${ }^{24}$ the prospective relationship at 2 and 4 years of follow-up between frailty and incident depression was significant in adjusted models ( $\mathrm{OR}=3.75 ; 95 \% \mathrm{CI}=1.07-13.16)$, in adults aged $\geq 55$ years without baseline depressive symptomatology $(\mathrm{N}=1,827) .{ }^{24}$ Over a shorter time frame of 15 months of follow-up in older adults aged $\geq 65$ years $(\mathrm{N}=3,025)$ without baseline depressive symptomatology, a smaller percentage $(7.5 \%)$ developed depressive symptomatology, but frailty was also an independent predictor of incident depressive symptomatology in similarly adjusted models (OR $=1.86 ; 95 \%$ $\mathrm{CI}=1.05-3.28){ }^{29}$

\section{Frailty and depression as overlapping constructs}

A few studies examining the construct validity of frailty and depression using latent variables analyses have reported that they are "overlapping but distinct" constructs with $\sim 60 \%$ shared variance, and that "shared symptoms" between the biological syndromes of frailty and depression account for 0.23 of that correlation, ${ }^{21,22}$ however, these studies appear to have more liberal inclusion criteria (ie, participants with a history of clinical depression), and therefore it is unclear if dementia and stroke are part of the exclusion criteria as in the Fried et al's ${ }^{1}$ study. For example, Mezuk et al ${ }^{14}$ demonstrated that models of frailty and depression with differing numbers of classes (two in frailty; three in depression) that represent severity provided a better fit to their data, but that the constructs shared a substantial amount of variance (latent Kappa coefficient $=0.66$ ) and identified overlapping subgroups of participants. Using a confirmatory factor analytic approach in the $\mathrm{HRS}^{21}$ study in 3,453 participants, the same investigators demonstrated a moderate correlation $(0.68)$ between latent categorical models of depression and the "biological definition" of frailty, but when overlapping symptoms were accounted for this decreased to 0.45 . Although construct overlap between depression and frailty did not differ by sex, $73 \%$ of those severely depressed individuals were also categorized as concurrently frail. ${ }^{21}$

\section{Moderators of the depression and frailty relationship}

Studies that examine the moderating role of "other" factors on the depression and frailty relationship, in particular health, nutrition, and medication use, also provide a useful entry point into translating the findings in this review and optimizing the frailty construct as a clinically meaningful one. Although most, if not all, of the studies reviewed here use a covariate adjustment approach to examining - cross-sectional or longitudinal directional associations between the two constructs - a few have specifically examined moderators of depression on frailty. For example, Chang et al $^{23}$ examined cross-sectional interactions between comorbid disease pairs on frailty incidence in the Women's Health and Aging Studies $(\mathrm{N}=620)$ and found that frailty risk was increased in those participants with both depressive symptomatology and anemia, with the proportion of cases attributable to this disease pair at $56 \%$. The interaction between depressive symptomatology and weight has also been explored as a predictor of strength decline in frail elderly men over a 3-year follow-up period in the Honolulu Heart study, demonstrating greater declines in those who were depressed and underweight (body mass index $\left.[\mathrm{BMI}]<20 \mathrm{~kg} / \mathrm{m}^{2}\right),{ }^{30}$ although all frailty criteria were not measured. Similar interactions between depression and sarcopenia/osteopenia and depression and hip fracture were noted in a review ${ }^{31}$ highlighting potential intervention targets. Counterintuitive findings related to medication use and depressive symptomatology showed that antidepressant use doubled the risk of incident frailty in older women with depressive symptomatology, ${ }^{27}$ whereas low-potency statin users with a longer duration of use demonstrated a trend for a reduced risk of frailty, which was attenuated by inclusion of women with baseline use of antidepressants. ${ }^{28}$

\section{Discussion}

The findings of this systematic review suggest that more than one in ten older adults are either frail or have depressive symptomatology, and that a high percentage of older adults who are frail also have depressive symptomatology. Although the prevalence rates of both frailty and depression are highly variable across both cross-sectional and longitudinal studies at baseline $(2.5 \%-21.1 \%$ and $6.5 \%-25.3 \%$ for frailty and depressive symptomatology, respectively), due in large part to differences in the classification and nature of depressive symptomatology as well as the liberal inclusion criteria of a few studies that include dementia or stroke, incident frailty rates are less variable $(7.2 \%-18.6 \%)$ with most prevalence reports in the range of $13.6 \%-18.6 \%$. Incident depressive symptomatology is reported in very few of these studies to make generalizations. The coexistence of the two syndromes is also variable $(16.4 \%-53.8 \%)$ and infrequently reported at follow-up in longitudinal studies. Although there are a number of inconsistencies across the studies reported (we note that less than $5 \%$ of the articles screened for the review met the inclusion and exclusion criteria), the apparently 
moderate frequency of depressive symptomatology in frail older adults is concerning.

Although the reviewed studies used similar and adequate construct definitions of frailty and depressive symptomatology, their exclusion criteria varied from each other and from that of Fried et $\mathrm{al}^{1}$ in some cases, resulting in inconsistencies across study results. For example, the inclusion of cognitively impaired individuals appeared to inflate the odds of finding a frailty/depression relationship at an earlier, pre-frail stage in some cross-sectional studies. ${ }^{18,19}$ Similarly, studies reporting substantial shared variance between the biological syndromes of frailty and depression ${ }^{21,22}$ appear to have more liberal inclusion criteria (ie, participants with a history of clinical depression), and therefore it is unclear whether dementia and stroke are part of the exclusion criteria as in the Fried et al's ${ }^{1}$ study. Although the case has been made for including depression or depressive symptomatology as a part of the frailty criteria, it is also not clear whether they differentiate between "conditions that could potentially present with frailty characteristics as a consequence of a single disease" and older adults with a frailty "syndrome" per se, because they do not exclude the former in their models. In other words, those with a history of clinical depression, stroke, or dementia could be frail for different reasons than those without, which was the original point made by Fried et $\mathrm{al}^{1}{ }^{1}$ in their landmark study. Likely, it would continue to be clinically meaningful to rule out a history of clinical depression, or a history of stroke or dementia in frailty assessment, because the treatment approach might differ for older adults who are frail in the absence of the aforementioned symptoms.

Prospective studies of the relationship between depressive symptomatology and incident frailty provide the best evidence for increased frailty or depressive symptomatology risk. We examined studies looking at both the prospective relationship between depressive symptomatology and frailty and vice versa. The prospective relationship between depressive symptomatology and increased risk of incident frailty appeared to be the most robust, although most of the research has focused on this question. The prospective relationship between frailty and incident depressive symptomatology, although not as robust (with marginal findings in some reported studies), add to an emergent picture of the ways in which frailty may unfold over time in those who are, or are not, depressed in later life. Further research that examines the dynamic nature of these relationships might identify subgroups at greater risk for accelerated declines into a frailty state who would benefit from targeted interventions, such as hip-fractured older adults who become depressive and then frail. ${ }^{32}$ Studies such as these highlight the need to know more about the optimal timing of interventions for frail and/or depressive elderly.

Examining moderators of the relationship between depressive symptoms and frailty, specifically depressive symptom and disease pairs, is one systematic way to potentially design interventions that strategically target frailty in a clinically meaningful way. The studies reviewed here identified an increased concurrent risk of frailty in older adults with depressive symptomatology who had anemia, ${ }^{23}$ sarcopenia/osteopenia or hip fracture, ${ }^{31}$ greater risk of incident frailty in older adults with co-occurring depressive symptomatology and underweight (BMI $<20 \mathrm{~kg} / \mathrm{m}^{2}$ ), ${ }^{30}$ and antidepressant use. ${ }^{27}$ This type of methodological approach points to feasible and potentially low-cost nonpharmacologic interventions that target the depression and frailty relationship such as evaluating and improving nutritional status, establishing contraindications for prescribing antidepressants in the old and frail, and addressing the increased vulnerability of older adults with hip fracture alone and hip fracture co-occurring with depressive symptomatology to increased frailty risk. At the very least, these are areas in which follow-up studies that replicate and extend these findings in other cohorts could be conducted.

The moderating role of newer generation antidepressants in the depression-frailty relationship remains unclear. Lakey et $\mathrm{al}^{27}$ demonstrated an association between antidepressant treatment (regardless of the depression status) and increased incidence of frailty in older women. While antidepressants may have contributed to an increased frailty risk, it is also possible that antidepressant use may reflect a more severe, recurrent, or chronic form of depression. Lakey et $\mathrm{al}^{27}$ found that depressed women using antidepressants had chronic comorbidities and poor health, suggesting that an overlap of depressive symptoms with a comorbid disease might facilitate incident frailty, regardless of antidepressant use. It is also unclear whether all antidepressant classes carry a similar relationship with frailty. Decreased production of proinflammatory cytokines has been observed when depressed patients are treated with certain (but not all) antidepressants. ${ }^{33-35}$ A recent meta-analysis reported that antidepressants, specifically selective serotonin reuptake inhibitors, reduce inflammatory marker levels, suggesting anti-inflammatory properties of these medications. ${ }^{36}$ Randomized controlled depression trials are therefore essential to further elucidate the role of antidepressants in the relationship between depression and frailty. Alternatively, empirically supported psychotherapeutic interventions might offer 
advantages over antidepressants in some depressed, frail older adults, although the investigations using nonpharmacologic modalities in this subpopulation have not yet been conducted.

Late-life depression and frailty share several pathophysiologic mechanisms. One such overlapping mechanism is subclinical cerebrovascular disease. The vascular depression hypothesis postulates that altered mood regulation and cognitive dysfunction in the elderly are due to subclinical cerebrovascular ischemia that disrupts frontostriatal neural circuits. ${ }^{37}$ Lending support to this theory is the observation of increased prefrontal white matter hyperintensities in patients with late-life depression. ${ }^{38} \mathrm{Katz}^{39}$ hypothesized that if effects of white matter lesions on the brain are more anterior, the clinical presentation is likely to be late-life depression, whereas if the white matter hyperintensities are more posterior, it may manifest in the form of gait disturbances, weakness, and slowness, which are also indicators of frailty. Consistent with this theory, cardiovascular disease, increased white matter lesion burden, and stroke have been found to be associated with frailty in older adults. ${ }^{10}$ Others have further theorized that vascular depression is a prodrome of frailty. ${ }^{11}$

Growing evidence also supports the role of chronic inflammation as a causative mechanism of both depression and frailty among older adults. Several studies have identified a positive association between frailty and inflammatory cytokine interleukin 6 (IL-6), C-reactive protein, and tumor necrosis factor- $\alpha$ levels..$^{12,13,40-42}$ The elevations in these inflammatory markers suggest chronic, low-level activation of inflammatory and immune mechanisms in frail older individuals. Similarly, "inflammation hypothesis" in geriatric depression has been proposed, whereby inflammatory processes are thought to promote changes in the brain's neural systems which predispose some to develop geriatric depression. ${ }^{43}$ Among the proinflammatory cytokines, elevated IL-6 levels have consistently been associated with significant depressive symptoms ${ }^{44,45}$ as well as depressive disorders $^{46}$ in the elderly.

Hypothalamic-pituitary-adrenal axis (HPA) dysregulation of hormones has been proposed as another possible etiology of both depression and frailty syndromes in older adults. Low levels of adrenal androgen dehydroepiandrosterone sulfate and insulin-like growth factor 1are associated with frailty. ${ }^{47}$ Lower insulin-like growth factor 1 concentrations may be associated with prevalent late-life depression especially in women, but in one longitudinal study, no relationship with incident depression was observed..$^{48}$ Further, age-related depletion in testosterone is thought to be associated with declines in muscle mass in men, an important component that contributes to frailty syndrome. ${ }^{49}$ Interestingly, lower testosterone levels have also been demonstrated in older men with dysthymic disorder. ${ }^{50}$ In two epidemiologic studies, ${ }^{51,52}$ that is, the Women's Health and Aging and the KORA-age studies, dysregulated diurnal cortisol variations were associated with frailty as evidenced by lower morning and higher evening salivary cortisol levels. Late-life depression is also associated with both hyper- and hypocortisol states; ${ }^{53}$ such hyperactivity and hypoactivity of the HPA axis have also been reported in older persons with significant depressive symptoms. ${ }^{54}$ Bereaved older adults with depression may also have more dysregulated cortisol patterns, ${ }^{55}$ and a similar finding of cortisol dysregulation has been found in older adults who fracture a hip and later become depressed and frail. ${ }^{32}$ These findings suggest that neuroendocrine dysregulation might be a common underlying mechanism of both late-life depression and frailty, although additional studies are needed to further clarify if the hormonal abnormalities that are seen in these syndromes are similar or distinct.

Finally, accelerated cellular aging, as measured by telomere length (TL) shortening, might also be linked to depression and frailty, although studies have produced inconclusive results. TL was not related to the clinical phenotype of frailty in some studies, ${ }^{56,57}$ although it was shorter in older patients with sarcopenia. ${ }^{58}$ Similar contradictory findings are also reported in depression. Although shorter TL is reported in depressed adults, ${ }^{59,60}$ recent studies of late-life depression have found no evidence for accelerated cellular aging. ${ }^{61}$ A greater understanding of how these overlapping etiologic mechanisms contribute to both depression and frailty will advance the development of prevention strategies in at-risk groups and assist in targeting clinical interventions to older adults with these syndromes, either when they occur alone or coexist with each other, with the goal of preventing disability and premature death.

Although much of the interest in frailty is the potential reversibility of many of its features, well-designed intervention studies targeting both frailty and depression are rare or nonexistent. A few longitudinal studies have found that interventions involving physical activity may have an impact on individual physical health indicators of frailty in community dwelling older adults; however, these studies are limited because they do not report the frailty composite as an outcome or use the Fried et al' $s^{1}$ criteria, and rarely include depressive symptomatology, making comparisons across studies difficult. In a 4-month longitudinal pre- and 
post-intervention design, Wolf et $\mathrm{a}^{62}$ found less decline in grip strength for participants performing tai chi than balance training or education only, but a greater increase in walking distance in the balance training group, and did not measure depressive symptomatology. Likewise, participants (classified as frail using the Edmonson Frail Scale which includes items measuring cognitive impairment, functional ability, and mobility) of the HOPE trial were less likely to have a significant decline in the timed up and go test after participating in an exercise routine consisting of strengthening and physiotherapy than a control group, but the intervention had no influence on depressive symptomatology. ${ }^{63}$ Fusco et al found nondepressed participants classified as frail using the Minimum Data Set for Home Care (MDS-HC), who participated in a rehabilitation program for 3 hours per week for 6 months, were more likely to maintain or improve their functional status. ${ }^{64}$ Similarly, Kono et al ${ }^{65}$ found less decline in activities of daily living, instrumental activities of daily living, and depression in an intervention group that participated in a rehabilitation program for participants classified as frail using the Japanese long-term care insurance system. Physical activity may decrease rates of decline in physical function (often confounded with frailty in intervention studies) and depressive symptoms via inflammatory mechanisms, ${ }^{66}$ but it is unclear how safe these interventions might be in very frail individuals, who are usually not included in the intervention studies. Additional exploration is needed to determine the effectiveness of physical activity interventions in frail older adults.

Potential interventions that modulate the endocrine system (eg, anabolic hormones such as dehydroepiandrosterone sulfate and testosterone) or inflammatory processes may also be beneficial in treating or preventing depression and frailty in older adults. Exogenous testosterone administration is found to increase physical performance and strength in older testosterone-deficient men. ${ }^{67}$ Testosterone therapy is also shown to decrease depressive symptoms in males with late-life depression, especially in those with late-onset depression. ${ }^{68}$ Concerns, however, remain about the long-term safety of testosterone therapy (eg, risk of prostate neoplasia); therefore, studies that examine the risks and the benefits of such interventions need to be conducted. Emerging evidence also points to the therapeutic benefits of anti-inflammatory drugs in major depression, ${ }^{69-71}$ but studies including older adults are rare to none. Similarly, although observational studies using drugs with anti-inflammatory properties (eg, statins) have produced negative findings in frailty, ${ }^{28}$ there is a dearth of randomized-controlled studies that have examined the effectiveness of antioxidants and anti-inflammatory agents in older frail adults. Finally, aggressively comanaging vascular risk factors and depression may prevent development of frailty in the elderly. ${ }^{11}$

This systematic review identified studies that included the biological definition of the frailty syndrome ${ }^{1}$ and depressive symptomatology measured with standardized instruments, including cross-sectional, prospective longitudinal, and latent variable analyses of their overlapping construct validity. We examined the prevalence of incident frailty and depressive symptomatology and their co-occurrence in older adults above the age of 55 years. We note that a large percentage of the studies that we reviewed did not meet the inclusion criteria. Further, we identified moderating factors and common-cause mechanisms contributing to this relationship, and tried to identify intervention studies that target these relationships. Although we did not include studies in the review with other frailty definitions, we note that several good reviews have recently done this. ${ }^{6,7}$ Instead, our focus was on larger epidemiological studies with well-defined constructs, in order to minimize the methodological variance across the studies. ${ }^{15}$ Finally, it was challenging to disentangle the causal conundrum between depressive symptomatology and frailty with the currently available literature, which is observational in nature.

The incidence of depressive symptomatology or frailty or their co-occurrence was higher than $10 \%$ in older adults aged $\geq 55$ years and these prevalence rates varied widely, but less so in large epidemiological studies of new incident frailty. A few sources of this variability were identified by this review. These include the imprecise measurement of depressive symptomatology and confounding the frailty syndrome with single disease outcomes in older adults with severe depression, cognitive impairment, dementia, or stroke. These sources may account for a higher prevalence of pre-frailty or earlier frailty onset in some studies and for a substantial proportion of the shared variance in the depressive symptomatology and frailty constructs. Prospective studies of the relationship between depressive symptomatology and incident frailty provide good evidence for increased frailty and/or depressive symptomatology risk and highlight the need to know more about the optimal timing of interventions for frail and/or depressive elderly. Future investigations using multidisciplinary interventions that target depression and frailty are critically needed to determine whether such modalities will decrease or prevent long-term adverse outcomes such as falls, disability, and premature mortality in the elderly. 


\section{Disclosure}

The authors report no conflicts of interest in this work.

\section{References}

1. Fried LP, Tangen CM, Walston J, et al. Frailty in older adults: evidence for a phenotype. J Gerontol A Biol Sci Med Sci. 2001;56(3):M146-M157. doi:10.1093/gerona/56.3.M146.

2. Fried LP, Borhani NO, Enright P, et al. The cardiovascular health study: design and rationale. Ann Epidemiol. 1991;1(3):263-276. doi:10.1016/1047-2797(91)90005-W.

3. Rockwood K, Fox RA, Stolee P, Robertson D, Beattie BL. Frailty in elderly people: an evolving concept. CMAJ. 1994;150(4):489-495.

4. Strawbridge WJ, Shema SJ, Balfour JL, Higby HR, Kaplan GA. Antecedents of frailty over three decades in an older cohort. J Gerontol B Psychol Sci Soc Sci. 1998;53B(1):S9-S16. doi:10.1093/ geronb/53B.1.S9.

5. Cigolle CT, Ofstedal MB, Tian Z, Blaum CS. Comparing models of frailty: The Health and Retirement Study. J Am Geriatr Soc. 2009; 57(5):830-839. doi:10.1111/j.1532-5415.2009.02225.x.

6. Mezuk B, Edwards L, Lohman M, Choi M, Lapane K. Depression and frailty in later life: a synthetic review. Int J Geriatr Psychiatry. 2012;27(9):879-892. doi:10.1002/gps.2807.

7. Buigues C, Padilla-Sánchez C, Garrido JF, Navarro-Martínez R, Ruiz-Ros V, Cauli O. The relationship between depression and frailty syndrome: a systematic review. Aging Ment Health. 2015;19(9):762-772. doi:10.1080/13607863.2014.967174.

8. Bandeen-Roche K, Xue Q-L, Ferrucci L, et al. Phenotype of frailty: characterization in the women's health and aging studies. J Gerontol A Biol Sci Med Sci. 2006;61(3):262-266.

9. Xue Q-L, Bandeen-Roche K, Varadhan R, Zhou J, Fried LP. Initial manifestations of frailty criteria and the development of frailty phenotype in the Women's Health and Aging Study II. J Gerontol A Biol Sci Med Sci. 2008;63(9):984-990.

10. Newman AB, Gottdiener JS, McBurnie MA, et al. Associations of subclinical cardiovascular disease with frailty. J Gerontol A Biol Sci Med Sci. 2001;56(3):M158-M166. doi:10.1093/gerona/56.3.M158.

11. Paulson D, Lichtenberg PA. Vascular depression: an early warning sign of frailty. Aging Ment Health. 2013;17(1):85-93. doi:10.1080/13607863. 2012.692767.

12. Walston J, McBurnie M, Newman A, et al. Frailty and activation of the inflammation and coagulation systems with and without clinical comorbidities: results from the cardiovascular health study. Arch Intern Med. 2002;162(20):2333-2341. doi:10.1001/archinte.162. 20.2333.

13. Leng SX, Xue Q-L, Tian J, Walston JD, Fried LP. Inflammation and frailty in older women. $J$ Am Geriatr Soc. 2007;55(6):864-871. doi:10.1111/j.1532-5415.2007.01186.x.

14. Mezuk B, Lohman M, Dumenci L, Lapane KL. Are depression and frailty overlapping syndromes in mid- and late-life? A latent variable analysis. Am J Geriatr Psychiatry. 2013;21(6):560-569. doi:10.1016/j. jagp.2012.12.019.

15. Rodríguez-Mañas L, Féart C, Mann G, et al. Searching for an operational definition of frailty: a Delphi method based consensus statement. The Frailty Operative Definition-Consensus Conference Project. J Gerontol A Biol Sci Med Sci. 2013;68(1):62-67. doi:10.1093/gerona/gls119.

16. Radloff LS. The CES-D Scale. A self-report depression scale for research in the general population. Appl Psychol Meas. 1977;1(3):385-401. doi: $10.1177 / 014662167700100306$

17. Yesavage JA. Geriatric Depression Scale. Psychopharm Bull. 1988;24: 709-711.

18. Sánchez-García S, Sánchez-Arenas R, García-Peña C, et al. Frailty among community-dwelling elderly Mexican people: prevalence and association with sociodemographic characteristics, health state and the use of health services. Geriatr Gerontol Int. 2014;14(2):395-402. doi:10.1111/ggi.12114.
19. Jürschik P, Nunin C, Botigué T, Escobar MA, Lavedán A, Viladrosa M. Prevalence of frailty and factors associated with frailty in the elderly population of Lleida, Spain: the FRALLE survey. Arch Gerontol Geriatr. 2012;55(3):625-631. doi:10.1016/j.archger.2012.07.002.

20. Pegorari MS, Tavares DMDS. Factors associated with the frailty syndrome in elderly individuals living in the urban area. Rev Lat Am Enfermagem. 2014;22(5):874-882.

21. Lohman M, Dumenci L, Mezuk B. Sex differences in the construct overlap of frailty and depression: evidence from the Health and Retirement Study. J Am Geriatr Soc. 2014;62(3):500-505. doi:10.1111/jgs. 12689.

22. Lohman M, Dumenci L, Mezuk B. Depression and frailty in late life: evidence for a common vulnerability. J Gerontol B Psychol Sci Soc Sci. In press 2014.

23. Chang SS, Weiss CO, Xue Q-L, Fried LP. Patterns of comorbid inflammatory diseases in frail older women: the Women's Health and Aging Studies I and II. J Gerontol A Biol Sci Med Sci. 2010;65:407-413. doi:10.1093/gerona/glp181.

24. Feng L, Nyunt MSZ, Feng L, Yap KB, Ng TP. Frailty predicts new and persistent depressive symptoms among community-dwelling older adults: findings from Singapore longitudinal aging study. J Am Med Dir Assoc. 2014;15(1):76.e7-e76.e12. doi:10.1016/j.jamda.2013.10.001.

25. Collard RM, Comijs HC, Naarding P, et al. Frailty as a predictor of the incidence and course of depressed mood. J Am Med Dir Assoc. 2015; 16(5):570-578. doi:10.1080/13607863.2013.827628.

26. Woods NF, LaCroix AZ, Gray SL, et al; Women's Health Initiative. Frailty: emergence and consequences in women aged 65 and older in the Women's Health Initiative Observational Study. J Am Geriatr Soc. 2005;53(8):1321-1330. doi:10.1111/j.1532-5415.2005.53405.x.

27. Lakey SL, LaCroix AZ, Gray SL, et al. Antidepressant use, depressive symptoms, and incident frailty in women aged 65 and older from the Women's Health Initiative Observational Study. J Am Geriatr Soc. 2012;60(5):854-861. doi:10.1111/j.1532-5415.2012.03940.x.

28. LaCroix AZ, Gray SL, Aragaki A, et al. Statin use and incident frailty in women aged 65 years or older: prospective findings from the Women's Health Initiative Observational Study. J Gerontol A Biol Sci Med Sci. 2008;63(4):369-375.

29. Makizako H, Shimada H, Doi T, et al. Physical frailty predicts incident depressive symptoms in elderly people: prospective findings from the Obu Study of Health Promotion for the Elderly. J Am Med Dir Assoc. 2015;16(3):194-199. doi:10.1016/j.jamda.2014.08.017.

30. Rantanen T, Penninx BW, Masaki K, Lintunen T, Foley D, Guralnik JM. Depressed mood and body mass index as predictors of muscle strength decline in old men. J Am Geriatr Soc. 2000;48:613-617.

31. Rolland Y, Abellan van Kan G, Bénétos A, et al. Frailty, osteoporosis and hip fracture: causes, consequences and therapeutic perspectives. J Nutr Health Aging. 2008;12:335-346.

32. Phillips AC, Upton J, Duggal NA, Carroll D, Lord JM. Depression following hip fracture is associated with increased physical frailty in older adults: the role of the cortisol: dehydroepiandrosterone sulphate ratio. BMC Geriatr. 2013;13:60. doi:10.1186/1471-2318-13-60.

33. Kim Y-K, Na K-S, Shin K-H, Jung H-Y, Choi S-H, Kim J-B. Cytokine imbalance in the pathophysiology of major depressive disorder. Prog Neuropsychopharmacol Biol Psychiatry. 2007;31(5):1044-1053. doi:10.1016/j.pnpbp.2007.03.004.

34. Miller AH, Maletic V, Raison CL. Inflammation and its discontents: the role of cytokines in the pathophysiology of major depression. Biol Psychiatry. 2009;65(9):732-741. doi:10.1016/j.biopsych.2008.11.029.

35. Janssen DGA, Caniato RN, Verster JC, Baune BT. A psychoneuroimmunological review on cytokines involved in antidepressant treatment response. Hum Psychopharmacol Clin Exp. 2010;25(3):201-215. doi:10.1002/hup.1103.

36. Hannestad J, DellaGioia N, Bloch M. The effect of antidepressant medication treatment on serum levels of inflammatory cytokines: a meta-analysis. Neuropsychopharmacology. 2011;36(12):2452-2459. doi:10.1038/npp.2011.132. 
37. Taylor WD, Aizenstein HJ, Alexopoulos GS. The vascular depression hypothesis: mechanisms linking vascular disease with depression. Mol Psychiatry. 2013;18(9):963-974. doi:10.1038/mp.2013.20.

38. Herrmann LL, Masurier ML, Ebmeier KP. White matter hyperintensities in late life depression: a systematic review. J Neurol Neurosurg Psychiatry. 2008;79(6):619-624. doi:10.1136/jnnp.2007.124651.

39. Katz IR. Depression and frailty: the need for multidisciplinary research. Am J Geriatr Psychiatry. 2004;12(1):1-5. doi:10.1097/00019442200401000-00001.

40. Leng S, Chaves P, Koenig K, Walston J. Serum interleukin-6 and hemoglobin as physiological correlates in the geriatric syndrome of frailty: a pilot study. J Am Geriatr Soc. 2002;50(7):1268-1271. doi:10.1046/j.1532-5415.2002.50315.x.

41. Hubbard RE, O’Mahony MS, Savva GM, Calver BL, Woodhouse KW. Inflammation and frailty measures in older people. $J$ Cell Mol Med 2009;13(9b):3103-3109. doi:10.1111/j.1582-4934.2009.00733.x.

42. Fernández-Garrido J,Ruiz-Ros V, Buigues C,Navarro-MartinezR, CauliO. Clinical features of prefrail older individuals and emerging peripheral biomarkers: a systematic review. Arch Gerontol Geriatr. 2014;59(1) 7-17. doi:10.1016/j.archger.2014.02.008.

43. Alexopoulos GS, Morimoto SS. The inflammation hypothesis in geriatric depression. Int J Geriatr Psychiatry. 2011;26(11):1109-1118. doi:10.1002/gps.2672.

44. Bremmer MA, Beekman ATF, Deeg DJH, et al. Inflammatory markers in late-life depression: results from a population-based study. $J$ Affect Disord. 2008;106(3):249-255. doi:10.1016/j.jad.2007.07.002.

45. Penninx BWJH, Kritchevsky SB, Yaffe K, et al. Inflammatory markers and depressed mood in older persons: results from the health, aging and body composition study. Biol Psychiatry. 2003;54(5):566-572 doi:10.1016/S0006-3223(02)01811-5.

46. Tiemeier H, Hofman A, van Tuiji HR, Kiliaan AJ, Meijer J, Breteler MM. Inflammatory proteins and depression in the elderly. Epidemiology. 2003;14(1):103-107.

47. Leng SX, Cappola AR, Andersen RE, et al. Serum levels of insulin-like growth factor-I (IGF-I) and dehydroepiandrosterone sulfate (DHEA-S), and their relationships with serum interleukin-6, in the geriatric syndrome of frailty. Aging Clin Exp Res. 2004;16(2):153-157. doi:10.1007/ BF03324545.

48. van Varsseveld NC, van Bunderen CC, Sohl E, et al. Serum insulinlike growth factor 1 and late-life depression: a population-based study. Psychoneuroendocrinology. 2015;54:31-40. doi:10.1016/j. psyneuen.2015.01.014.

49. Muller M, Grobbee DE, Thijssen JHH, van den Beld AW, van der Schouw YT. Sex hormones and male health: effects on components of the frailty syndrome. Trends Endocrinol Metab. 2003;14(6):289-296. doi:10.1016/S1043-2760(03)00083-3.

50. Seidman SN, Araujo AB, Roose SP, et al. Low testosterone levels in elderly men with dysthymic disorder. Am J Psychiatry. 2002;159(3): 456-459. doi:10.1176/appi.ajp.159.3.456.

51. Varadhan R, Walston J, Cappola AR, Carlson MC, Wand GS, Fried LP. Higher levels and blunted diurnal variation of cortisol in frail older women. J Gerontol A Biol Sci Med Sci. 2008;63(2):190-195.

52. Johar H, Emeny RT, Bidlingmaier M, et al. Blunted diurnal cortisol pattern is associated with frailty: a cross-sectional study of 745 participants aged 65 to 90 Years. J Clin Endocrinol Metab. 2014;99(3):E464-E468. doi:10.1210/jc.2013-3079.

53. Bremmer MA, Deeg DJH, Beekman ATF, Penninx BWJH, Lips P, Hoogendijk WJG. Major depression in late life is associated with both hypo- and hypercortisolemia. Biol Psychiatry. 2007;62(5):479-486. doi:10.1016/j.biopsych.2006.11.033.

54. Penninx BWJH, Beekman ATF, Corsi AM, et al. Late-life depressive symptoms are associated with both hyperactivity and hypoactivity of the hypothalamo-pituitary-adrenal axis. Am J Geriatr Psychiatry. 2007; 15(6):522-529. doi:10.1097/JGP.0b013e318033ed80.

55. Holland JM, Thompson KL, Rozalski V, Lichtenthal WG. Bereavementrelated regret trajectories among widowed older adults. $J$ Gerontol $B$ Psychol Sci Soc Sci. 2014;69B(1):40-47. doi:10.1093/geronb/gbt050.
56. Brault ME, Ohayon SM, Kwan R, et al. Telomere length and the clinical phenotype of frailty in older adults undergoing cardiac surgery. $J \mathrm{Am}$ Geriatr Soc. 2014;62(11):2205-2207. doi:10.1111/jgs.13076.

57. Saum K-U, Dieffenbach AK, Müezzinler A, et al. Frailty and telomere length: cross-sectional analysis in 3537 older adults from the ESTHER cohort. Exp Gerontol. 2014;58:250-255. doi:10.1016/j. exger.2014.08.009.

58. Marzetti E, Lorenzi M, Antocicco M, et al. Shorter telomeres in peripheral blood mononuclear cells from older persons with sarcopenia: results from an exploratory study. Front Aging Neurosci. 2014;6:233. doi:10.3389/fnagi.2014.00233.

59. Wolkowitz OM, Mellon SH, Epel ES, et al. Leukocyte telomere length in major depression: correlations with chronicity, inflammation and oxidative stress - preliminary findings. PLoS One. 2011;6(3):e17837. doi:10.1371/journal.pone.0017837.

60. Verhoeven JE, Révész D, Epel ES, Lin J, Wolkowitz OM, Penninx BWJH. Major depressive disorder and accelerated cellular aging: results from a large psychiatric cohort study. Mol Psychiatry. 2014;19(8): 895-901. doi:10.1038/mp.2013.151.

61. Schaakxs R, Verhoeven JE, Oude Voshaar RC, Comijs HC, Penninx BWJH. Leukocyte telomere length and late-life depression. Am J Geriatr Psychiatry. 2015;23(4):423-432. doi:10.1016/j.jagp. 2014.06.003.

62. Wolf SL, Barnhart HX, Kutner NG, McNeely E, Coogler C, Xu T; Atlanta FICSIT Group. Selected as the best paper in the 1990s: Reducing frailty and falls in older persons: an investigation of tai chi and computerized balance training. J Am Geriatr Soc. 2003;51:1794-1803.

63. Clegg A, Barber S, Young J, Iliffe S, Forster A. The Home-based Older People's Exercise (HOPE) trial: a pilot randomised controlled trial of a home-based exercise intervention for older people with frailty. Age Ageing. 2014;43:687-695. doi:10.1093/ageing/afu033.

64. Fusco D, Bochicchio GB, Onder G, Barillaro C, Bernabei R, Landi F; SILVERNET-HC Study Group of Basilicata Region. Predictors of rehabilitation outcome among frail elderly patients living in the community. J Am Med Dir Assoc. 2009;10:335-341. doi:10.1016/j. jamda.2009.02.004.

65. Kono A, Fujita T, Tsumura C, Kondo T, Kushiyama K, Rubenstein LZ. Preventive home visit model targeted to specific care needs of ambulatory frail elders: preliminary report of a randomized trial design. Aging Clin Exp Res. 2009;21:167-173.

66. Landi F, Abbatecola AM, Provinciali M, et al. Moving against frailty: does physical activity matter? Biogerontology. 2010;11:537-545. doi:10. 1007/s10522-010-9296-1.

67. Page ST, Amory JK, Bowman FD, et al. Exogenous testosterone (T) alone or with finasteride increases physical performance, grip strength, and lean body mass in older men with low serum T. J Clin Endocrinol Metab. 2005;90(3):1502-1510. doi:10.1210/jc.2004-1933.

68. Perry PJ, Yates WR, Williams RD, Andersen AE, MacIndoe JH, Holman TL. Testosterone therapy in late-life major depression in males. J Clin Psychiatry. 2002;63(12):1096-1101.

69. Müller N, Schwarz MJ, Dehning S, et al. The cyclooxygenase-2 inhibitor celecoxib has therapeutic effects in major depression: results of a doubleblind, randomized, placebo controlled, add-on pilot study to reboxetine. Mol Psychiatry. 2006;11(7):680-684. doi:10.1038/sj.mp.4001805.

70. Tyring S, Gottlieb A, Papp K, et al. Etanercept and clinical outcomes, fatigue, and depression in psoriasis: double-blind placebocontrolled randomised phase III trial. Lancet. 2006;367(9504):29-35. doi:10.1016/S0140-6736(05)67763-X.

71. Raison CL, Rutherford RE, Woolwine BJ, et al. A randomized controlled trial of the tumor necrosis factor-alpha antagonist infliximab in treatment resistant depression: role of baseline inflammatory biomarkers. JAMA Psychiatry. 2013;70(1):31-41. doi:10.1001/2013. jamapsychiatry.4. 


\section{Publish your work in this journal}

Clinical Interventions in Aging is an international, peer-reviewed journal focusing on evidence-based reports on the value or lack thereof of treatments intended to prevent or delay the onset of maladaptive correlates of aging in human beings. This journal is indexed on PubMed Central, MedLine,

CAS, Scopus and the Elsevier Bibliographic databases. The manuscript management system is completely online and includes a very quick and fair peer-review system, which is all easy to use. Visit http://www.dovepress. com/testimonials.php to read real quotes from published authors. 